\title{
Growth of hydrophobic membrane on copper surface and study of its corrosive behavior
}

\author{
Aikaterini Baxevani ${ }^{1}$, Fani Stergioudi ${ }^{1,}{ }^{*}$, Azarias Mavropoulos ${ }^{1}$, Nikolaos Michailidis ${ }^{1}$ \\ ${ }^{1}$ Physical Metallurgy Lab, School of Mechanical Engineering, Aristotle University of Thessaloniki, \\ GR541 24 Thessaloniki, Greece
}

\begin{abstract}
Hydrophobic films were produced on a copper surface by chemical methods. The whole process consisted of three stages. Initially the hierarchical structure of the surface was achieved by in situ reduction of $\mathrm{Ag}^{+}$ions to $\mathrm{Ag}$ using silver nitrate solution, thereby creating a nanostructured surface. The copper surface was then coated with polydopamine by a simple immersion procedure. Finally, it was decorated with thiols, to achieve a hydrophobic surface. The wetting angle was used to evaluate the hydrophobicity of the produced surfaces. The anti-corrosion behavior of the produced hydrophobic films, were evaluated on the specimens that showed higher hydrophobicity by electrochemical methods. Electrochemical techniques (potentiodynamic polarization and resistance spectroscopy tests) were performed in $3.5 \% \mathrm{NaCl}$ solution. Morphology was investigated by scanning electron microscopy (SEM). All experimental results showed a clear improvement in the corrosion behavior of the hydrophobic copper surfaces while the hydrophobicity was maintained after the corrosion tests.
\end{abstract}

\section{Introduction}

In recent years a plethora of metallic surfaces with special wetting ability have been manufactured through a combination of surface micro- and nanostructures and specific chemical composition, inspired by structures that exhibit materials of nature. Some of the mostly known water-repellent natural surfaces, are the lotus leaf, the shark skin and the skin of some insects. These metal surfaces with tailored wettability, are used in significant applications in corrosion protection systems, such as organic-water separation, fluid transport, self cleaning and anti-icing surfaces.

The wettability could be characterized by the contact angle (CA), between the surface and the droplet. When the CA of a surface is lower than $90^{\circ}$, the surface is described as a hydrophilic surface. When the CA is between $90^{\circ}$ and $150^{\circ}$, the surface is described as a hydrophobic surface and for contact angles more than $150^{\circ}$, the surface is superhydrophobic. For rough surfaces, the wetting state can be described by the Wenzel and Cassie-Baxter models.

\footnotetext{
*Corresponding author: fstergio@auth.gr
} 
There are various coating methods to produce super-hydrophobic surfaces that can be categorized as: i) rough surface fabrication with low surface energy material and ii) rough surface modification with low surface energy material [1,2]. The most well-known methods for developing hydrophobic surfaces are sol-gel, electrospining, layer-by-layer, plasma spray coating. These methods, although widely used, have many disadvantages. Typical is the sol-gel method, which involves multiple and complex steps, the electrospinning method, which requires specialized equipment, consumes high amounts of energy and can be produced only one specific nanostructure (fibers). Special equipment is also required in the plasma spray method while the spin coating method is unfriendly due to the use of environmentally harmful solvents $[3,4]$. Another method which is widely used, in order to create hydrophobic surfaces, is the immersion method. In the literature, this method either involve multiple steps, or require long residence times in solutions, or even if they meet the criteria of the simple and short method, the coatings produced do not have satisfactory mechanical stability [5-8].

In this research work, hydrophobic films were developed on copper sheets used as substrate due to its excellent properties (low weight, electrical conductivity) by a simple immersion method and with short immersion durations in solutions. The hydrophobic surfaces produced showed very good resistance to aqueous corrosive sodium chloride $(\mathrm{NaCl})$.

\section{Experimental methodology}

Copper sheets $(20 \times 20 \mathrm{~mm})$ were used as substrate. Before coating, the substrates were mechanically polished down to 1200 grit size using abrasive $\mathrm{SiC}$ papers. The copper substrates were then ultrasonically cleaned in acetone solution for $30 \mathrm{~min}$ for degreasing, followed by oxide removal by immersion in $0.1 \mathrm{M} \mathrm{HCl}$ for $5 \mathrm{~min}$ and subsequently rinsing in water and ethanol. The cleaned substrates were immersed in the silver nitrate $\left(\mathrm{AgNO}_{3}\right)$ solution $(4 \mathrm{mM})$ for durations of $5,10,15,20,30,40$ and 50 minutes, in a temperature of $30^{\circ} \mathrm{C}$ while stirring at $300 \mathrm{rpm}$.

To achieve a coherent and uniform silver (Ag) coating, a polydopamine aqueous solution (PDA) was used. The production of polydopamine was as follows: dopamine (DOPA) $(45 \mathrm{Mm})$, was added to a Tris-buffer solution which was prepared by dissolving $10 \mathrm{mM}$ trishydroxymethyl-aminomethane $\left(\mathrm{C}_{4} \mathrm{H}_{11} \mathrm{NO}_{3}\right)$ in distilled water. The obtained $\mathrm{pH}$ of the solution was measured to $\mathrm{pH}=10$. Samples were immersed for $20 \mathrm{~min}$ in the polydopamine aqueous solution, at a temperature of $40^{\circ} \mathrm{C}$ while the solution was constantly stirred at $300 \mathrm{rpm}$. Finally, for surface modification and reduction of surface energy, all specimens were immersed in an ethanolic solution of dodecanethiol (DDT) (0.1 M) for 1 hour.

The wetting characteristics of the sample surfaces were carried out by measuring static water contact angles. A water droplet of about $100 \mu \mathrm{l}$ was used for each measurement.

The microstructure of the films developed, was examined by scanning electron microscopy (SEM). The corrosion behavior of the coated copper specimens that showed better hydrophobicity (at the initial characterization via contact angle measurement) was tested in $3.5 \% \mathrm{w} / \mathrm{v} \mathrm{NaCl}$ aqueous solution by electrochemical techniques (potentiodynamic polarization and resistance spectroscopy (EIS) tests). Uncoated copper samples were also studied for reasons of comparison. All electrochemical experiments were performed in a three-electrode cell containing 3.5\% w/v aqueous $\mathrm{NaCl}$, according to ASTM D1193 IV. A platinum wire was used as an auxiliary electrode. The experimental installation of the electrochemical corrosion apparatus was in accordance with ASTM G 69-97 and ASTM 
G71-81. All electrochemical measurements were performed at room temperature $\left(25^{\circ} \mathrm{C}\right)$. In the case of polarization, the potential scan rate was $0.1 \mathrm{mV} / \mathrm{sec}$ and the scan range was -800 to $600 \mathrm{mV}$ relative to the reference electrode. Prior to the measurements of the polarization curves and the EIS, the working electrode was immersed in the test solution in open circuit (OCP) for 30 min to achieve steady state. The EIS was performed in the $0.01 \mathrm{~Hz}-100 \mathrm{kHz}$ frequency range using peak-to-peak voltage of $10 \mathrm{mV}$.

\section{Results and Discussion}

\subsection{Hydrophobicity and structural characterization}

The contact angle between a water droplet of about $100 \mu \mathrm{l}$ and the surface of the coated copper, with a thin layer of silver, for different immersion times in the silver nitrate solution $\left(\mathrm{AgNO}_{3}\right)$ is shown in Figure1. Each value is an average of 10 measurements. The contact angle exhibits superhydrophobic behavior and maximum contact angle of $153^{\circ}$ for a $15 \mathrm{~min}$ immersion time, in the silver nitrate solution. Figure $1 \mathrm{~b}$ shows typical images of water droplets of about $100 \mu \mathrm{l}$ on the surface of silver-coated copper corresponding to different times of growth of the silver films.

(a)

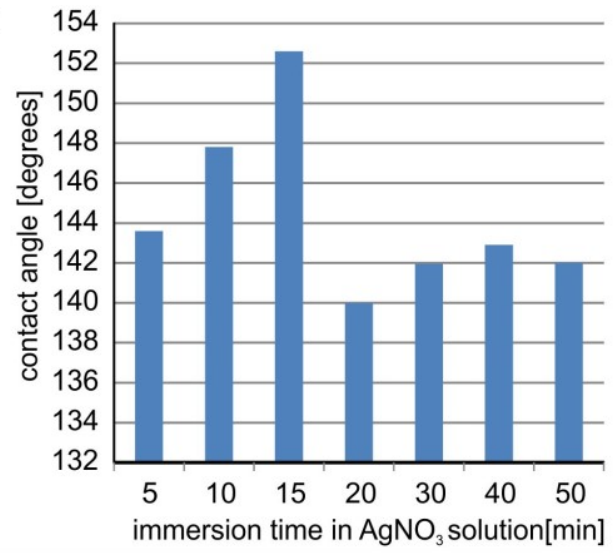

(c)

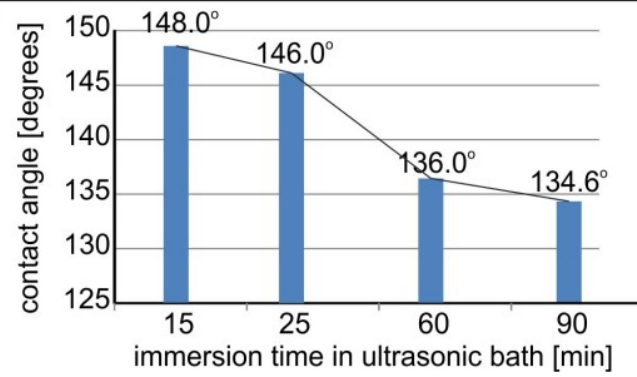

(b)
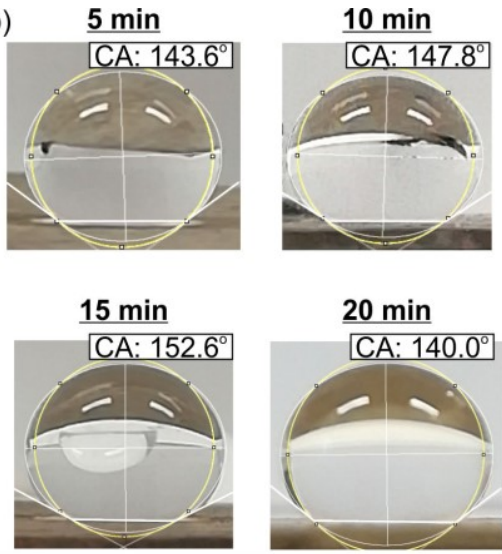

(d)
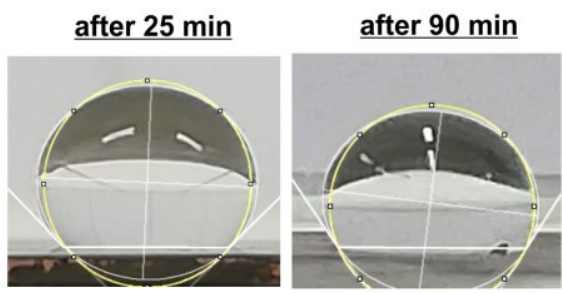

Fig. 1. (a) Contact angle relative to the immersion time in silver nitrate solution, (b) contact angle after various times in an ultrasonic bath for the optimal coated superhydrophpobic sample (15 min), (c) images with water droplets of about $60 \mu \mathrm{l}$ on the surface of silver-coated copper for various times of hydrophobic silver films growth, (d) images with water droplets of about $60 \mu 1$ on the surface of silver-coated copper after 25 and $90 \mathrm{~min}$ in an ultrasonic bath. 
For the initial testing of silver coating stability, the optimally coated samples (15 min) were immersed in an ultrasonic bath for various times and the probability of their hydrophobicity loss was investigated by measuring the contact angle as well as the possible abruption of the hydrophobic film. Figure 1c. shows that the contact angle decreases with increasing the immersion time in an ultrasonic bath. However, the hydrophobicity remains after 60 minutes and the contact angle remains almost constant at about $122^{\circ}$. It is noteworthy that no coating abruption was observed at any point on the coated specimens after 90 minutes in an ultrasonic bath. The results showed that there was a satisfactory stability and affinity of the developed silver films to the copper substrate.

The microstructure of the developed hydrophobic films is shown in Figure 2. A copper substrate in which the hydrophobic silver coatings were developed is also presented, for comparison. When the immersion time in the silver nitrate solution is $5 \mathrm{~min}$, the coating shows discontinuities, as shown in the SEM image and the copper substrate. By increasing the immersion time to $10 \mathrm{~min}$, the self-organization of silver ions and the development of needle-like nanostructures are observed. There are several clusters (of various sizes) so that the surface exhibits morphologically sufficient heterogeneity.
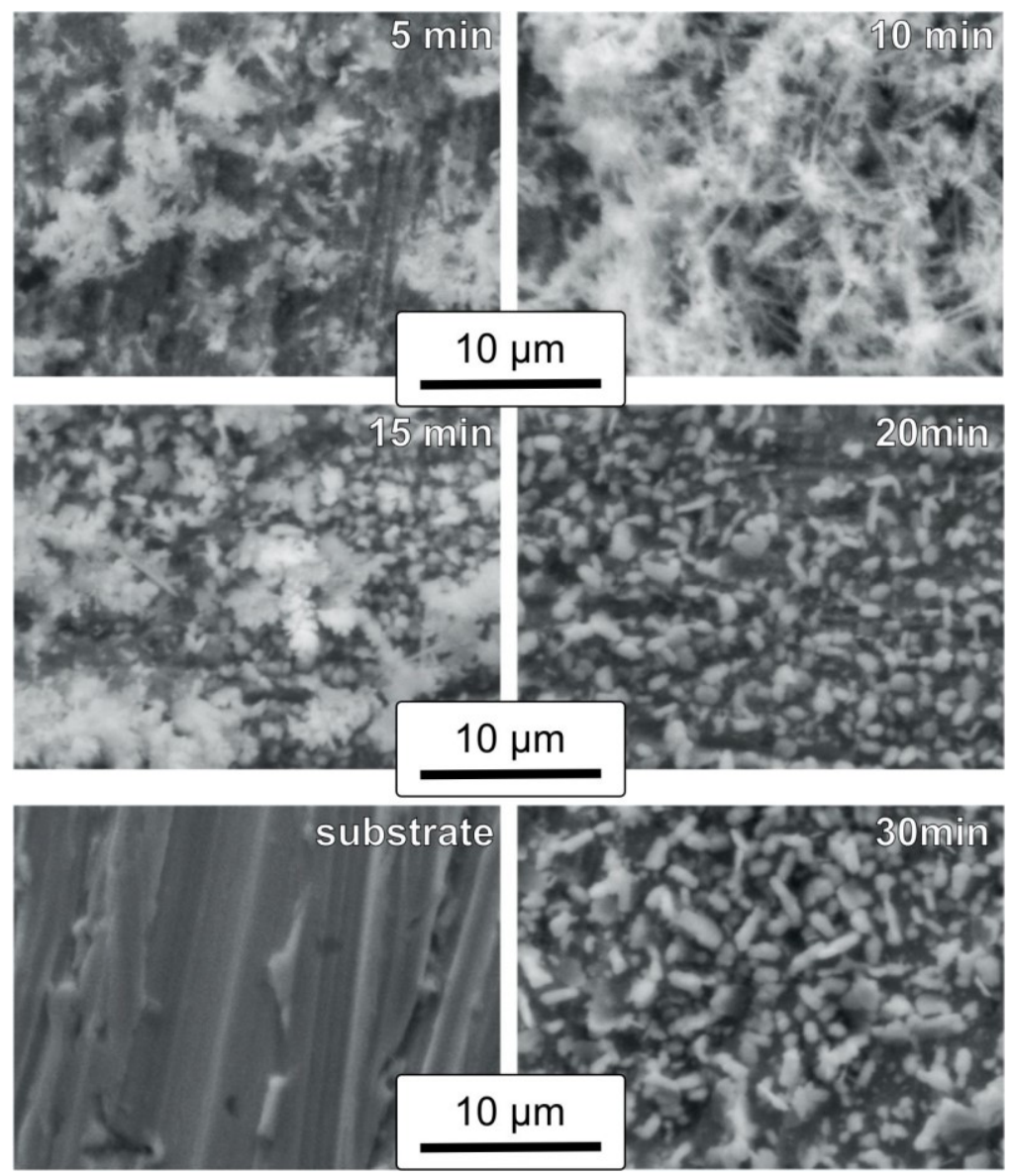

Fig. 2. (a) SEM images of hydrophobic films for different immersion times in silver nitrate solution 
Increasing the immersion time to $15 \mathrm{~min}$, has as a result the breakdown of agglomerates and the creation of new structural morphologies. Needle-like morphologies are now scarce and the sample exhibits dendritic or leaf-like morphologies. In addition, a second morphology of spherical agglomerates of approximately $1 \mu \mathrm{m}$ is shown. As the immersion time in the silver nitrate solution increases to $20 \mathrm{~min}$, the leaf-like structures decay and larger clusterls of silver with polyhedral morphology are appeared. This morphology (polyhedral aggregates) remains for $30 \mathrm{~min}$ in silver nitrate solution. The homogeneity and uniformity of the coating are excellent for immersion times greater than 20 min. However, due to the polyhedral morphology and relatively large (but uniform) aggregates the hydrophobicity decreases with the contact angle ranging from $140-143^{\circ}$.

\subsection{Corrosion behavior}

Figure 3 indicates that the character of the anodic and cathodic polarization curve for the silver-coated copper, is similar to that of the copper substrate, which indicates that the reactions, which occur in the two samples are the same. The same figure shows the values of corrosion potential and current. The hydrophobic silver layer, which was developed on the copper substrate, shifted the anodic and cathodic polarization curve to lower current densities, therefore increasing the corrosion resistance. It is worth noting that the hydrophobicity of the samples was maintained at a contact angle of $142^{\circ}$.

Figure 4 shows the Nyquist and Bode diagrams from EIS measurements. As observed, the copper substrate had a value of $/ \mathrm{Z} /=6 \mathrm{ohm} \mathrm{cm}^{2}$, at a high frequency of $10^{4} \mathrm{~Hz}$. However, the coated copper showed a value of $/ Z /=21 \mathrm{ohm} \mathrm{cm}^{2}$ which is almost 4 times higher than that of the substrate, on the same frequency. Similarly, at the low frequency of $0.01 \mathrm{~Hz}$, the /Z/ value of the copper substrate is $1.4 \mathrm{kohm} \mathrm{cm}^{2}$, while that of the coated copper is $7 \mathrm{kohm}$ $\mathrm{cm} 2$. The high value of the composite impedance $/ \mathrm{Z} /$ of the hydrophobic coating, compared to that of the copper substrate, indicates that the developed hydrophobic films are more corrosion resistant. The Nyquist diagrams show higher ohmic and capacitive resistance (real and imaginary part respectively) for the hydrophobic silver film.

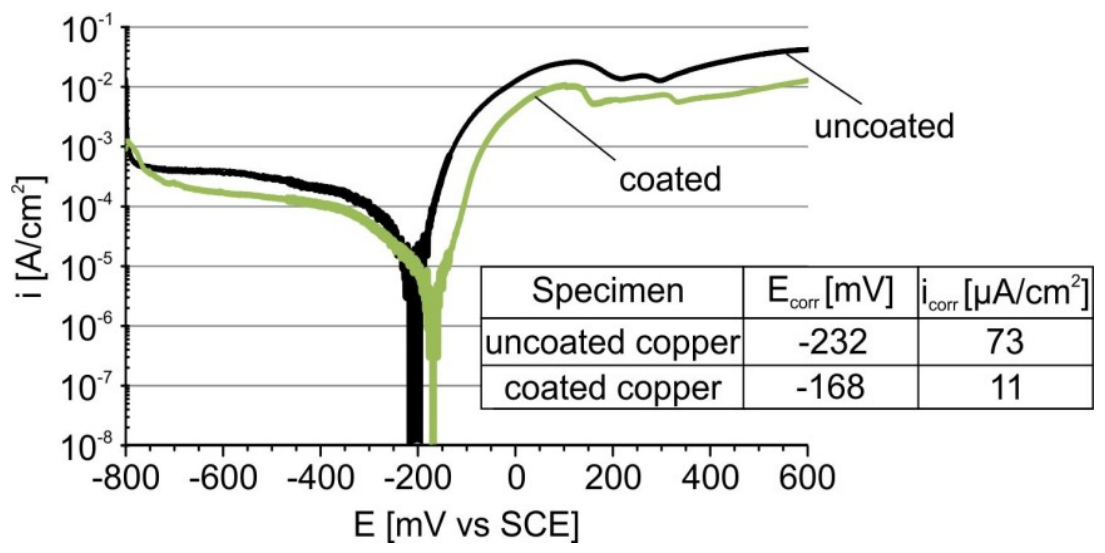

Fig. 3. Polarization curves for the silver-coated copper and uncoated copper. 
(a)

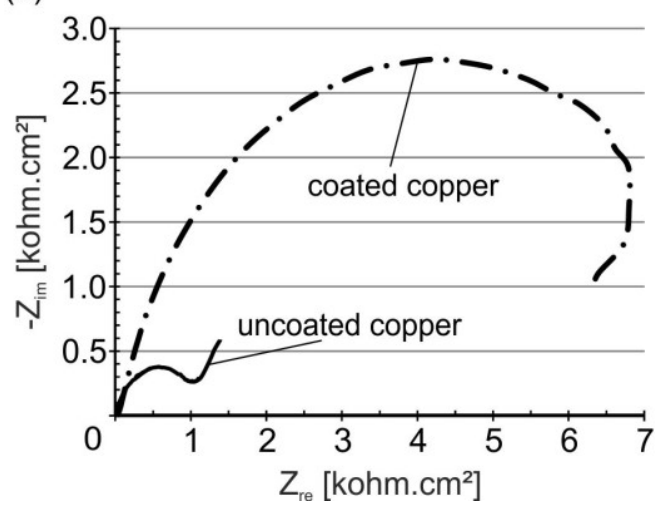

(b)

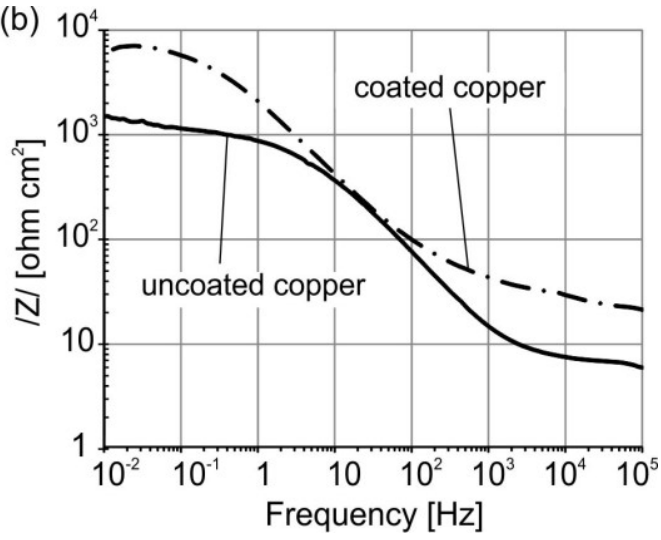

Fig. 4. (a) Nyquist and (b) Bode diagrams for silver coated copper and for copper substrate in 3.5 wt.\% aqueous solution $\mathrm{NaCl}$ at temperature of $25^{\circ} \mathrm{C}$.

\section{Conclusions}

Hydrophobic coatings were grown on a copper surface by chemical methods. The whole methodology is fast and simple, non-time-consuming and low-cost. By adjusting the immersion time in the silver nitrate solution, silver films of varying morphologies were studied which were studied for their hydrophobicity. Optimum contact angle at $152.6^{\circ}$ was achieved for a dip time of $15 \mathrm{~min}$. In this case the uniformity of the coating topology was achieved while its morphology reveals feather-like nanostructures. Developed hydrophobic silver film improves the corrosion resistance of copper thus demonstrating the multifunctionality of the developed nanostructured films.

\section{References}

1. A.M.A. A.M. Mohamed, Abdullah, N.A. Younan, Arab. J. Chem. 8, 749 (2015).

2. Gh.B. Darband, M. Aliofkhazraei, S. Khorsand, S. Sokhanvar, A. Kaboli., Arab. J. Chem., DOI: 10.1016/j.arabjc.2018.01.013.

3. Y. Chen, Sh. Yang, Q. Zhang, D. Zhang, Ch. Yang, Z. Wang, R. Wang, R. Song, W. Wang, Y. Zhao, Coatings 9, 69 (2019).

4. P. Varshney, S.S. Mohapatra, A. Kumar, Intern. J. Smart Nano Mater. 7, 248 (2016).

5. H.M. Ali, M.A. Qasim, S. Malik, G. Murtaza, Techniques for the Fabrication of SuperHydrophobic Surfaces and Their Heat Transfer Applications. Heat Transfer - Models, Methods and Applications (Kingston University London, London, England, 2018)

6. P. Che, W. Liu, X. Chang, A. Wang, Y. Han, Nano Research, DOI 10.1007/s12274015-0925-5 (2015).

7. D.K. Sarkar, N. Saleema, Surf. Coat. Techn. 15, 2483 (2010).

8. K. Liu, L. Jiang, Nanoscale 3, 825 (2011). 